\title{
BOBBLE-HEAD DOLL AND DANDY-WALKER SYNDROMES
}

A female infant with macrocephaly (head circumference $>95^{\text {th }} \%$ ), hydrocephalus, and Dandy-Walker syndrome, who developed horizontal head movements of the 'no-no' type at 1 year of age, is reported from Federal University of Minas Gerais, and other centers in Brazil. CT scan on day 1 of life showed a posterior fossa cyst, hypoplasia of the cerebellar vermis, elevation of the occipital lobe, and an increase in size of the posterior fossa, findings compatible with the Dandy-Walker syndrome. With progressive enlargement of the head, a VP shunt was inserted on day 9. She developed normally in the first year and walked at 14 months. The head circumference was at the $5^{\text {th }}$ percentile, and an MRI confirmed the cerebellar vermis hypoplasia and cyst, without signs of compression, enlargement or displacement of third or fourth ventricles or brainstem. Withdrawal of fluid from the fourth ventricle resulted in no change of the doll's head movements. At 3 year follow-up, head movements persisted, but the neurological examination and milestones of development were otherwise normal. Movements were worse when walking or with stress and they disappeared in sleep. (Henriques JG de B, Henriques KSW, Filho GP, Fonseca LF, Cardoso F, Da Silva MC. Bobble-head doll syndrome associated with Dandy-Walker syndrome. J Neurosurg (3 Suppl Pediatrics) Sept 2007;107:248-250). (Reprints: Jose Gilberto de Brito Henriques MD, email: henriques-jgb@hotmail.com )

COMMENT. The case-report suggests that bobble-head doll syndrome (BHDS) may occur with cerebellar malformation, and hydrocephalus with third ventricle dilatation is not an essential causative factor. Movements are usually up and down ('yes-yes' type), and the "no-no (side-to-side) movements seen in this case are rarely reported. The most common cause is a tumor or cyst and dilatation of the third ventricle, and movements often cease after ventricular drainage. Other causes include suprasellar arachnoid cyst, and aqueductal stenosis with hydrocephalus. Age of onset varies from 2 months to 13 years. The mechanisms suggested for BHDS include pressure on the medial aspect of the dorsomedial thalamic nucleus, intermittent obstruction of the formaen of Monro, or compression of the red nucleus. Malformation of the cerebellum, especially involving the vermis, may also be involved.

\section{INFANT DEVELOPMENTAL MILESTONES AND COGNITIVE OUTCOME}

The relationship between infant developmental milestones and later intellectual function was determined in a representative sample of 5,362 children born in the United Kingdom in 1946, after the second world war, and followed in to adulthood by researchers at the Behavioral and Clinical Neuroscience Institute, University of Cambridge; and University College London, UK. When participants reached 2 years of age, mothers were asked about ages of standing and walking, and saying words. Information on at least one cognitive test (IQ at age 8 , reading comprehension at age 26, or verbal fluency at age 53) and developmental variables was available in 3,969 subjects. Linear modeling demonstrated that earlier motor and speech development were significantly associated with greater IQ at age 8, higher reading compehension at age 26 , and better verbal fluency at age 57 . For every month earlier in learning to stand, the individual gained one half of one IQ point at age 8. Earlier development in speech and motor milestones was also associated with better reading 
comprehension at age 26 , but not at age 53 . The effect on reading was not solely driven by late developers. Speech development was related to reading comprehension at age 26 even when late developers were excluded. Later speech developers were less likely to progress beyond basic education. (Murray GK, Jones PB, Kuh D, Richards M. Infant developmental milestones and subsequent cognitive function. Ann Neurol August 2007;62:128-136). (Respond: Dr Graham K Murray, Brain Mapping Unit, Department of Psychiatry, University of Cambridge, c/o Experimental Psychology, Downing Street, Cambridge CB2 3EB, UK. Email:gm285@cam.ac.uk).

COMMENT. Later development of motor milestones and speech is associated with a small but significant impairment of intellectual function and educational attainment. The authors postulate a connection between delayed development of cortical-subcortical circuits in infancy and later cognitive dysfunction. A correlation between severe delay in developmental milestones and subsequent learning disability or mental retardation is well documented (von Wendt L et al. J Ment Defic Res 1984;28:219-225). In contrast, in the normal population it has been assumed that mild to moderate modifications of motor and speech developmental milestones are not related to later intellectual function. In a recent longitudinal birth cohort study by the Cambridge group of a Finnish population-based sample, it was found that a more rapid infant motor development is linked to better school performance and higher adult IQ scores. (Murray GK et al. J Child Psychol Psychiatry 2006;47:25-29). Frontal cortex, basal ganglia, and cerebellum volume is linearly related to the speed of infant motor development, according to one recent report. (Ridler K et al. Proc Natl Acad Sci USA 2006;103:15651-15656). The importance of developmental studies in the prediction of later educational attainment, indicated by the above reports, is also suggested by earlier but smaller nonepidemiological samples cited by the authors. In one report of a primary care cohort of 213 children, the age of learning to walk (a milestone most frequently remembered by a parent) was significantly associated with IQ at age 3 (Capute AJ et al. Clin Pediatr 1985;24:671-675).

\section{$\underline{\text { ATAXIC DISORDERS }}$}

\section{PRIMARY EPISODIC ATAXIAS}

The clinical and genetic diagnosis, genotype-phenotype correlations, pathophysiology and treatment of primary episodic ataxia syndromes are reviewed by researchers from Departments of Neurology, UCLA School of Medicine, Los Angeles, CA; National Hospital for Neurology, Queen Square, London, UK; Johns Hopkins University School of Medicine, Baltimore, MD; and University of Rochester School of Medicine, NY, USA. Primary episodic ataxias (EA) are autosomal dominant channelopathies that present with attacks of imbalance and incoordination. Six EA are described, but two (EA1 and EA2) affecting multiple families, with mutations in genes KCNA1 and CACNA1A, account for the majority of identified cases. Type 1 (EAl) is characterized by brief episodes of ataxia (seconds to minutes) and myokymia (or "neuromyotonia"), with onset in early childhood. Ataxia is precipitated by physical and emotional stress, startle or sudden movement. Attacks are associated with dysarthria and coarse tremor. Myokymia may be apparent clinically or only by EMG. Phenotypic variants can be associated with partial seizures, tight tendon Achilles, 